\title{
ATLAS on- $Z$ excess via gluino-Higgsino-singlino decay chains in the NMSSM
}

\author{
Keisuke Harigaya, ${ }^{a, b, c}$ Masahiro Ibe ${ }^{c, d}$ and Teppei Kitahara ${ }^{e, f, g}$ \\ ${ }^{a}$ Department of Physics, University of California, \\ Berkeley, California 94720, U.S.A. \\ ${ }^{b}$ Theoretical Physics Group, Lawrence Berkeley National Laboratory, \\ Berkeley, California 94720, U.S.A. \\ ${ }^{c}$ ICRR, University of Tokyo, \\ Kashiwa, Chiba 277-8582, Japan \\ ${ }^{d}$ Kavli IPMU (WPI), UTIAS, University of Tokyo, \\ Kashiwa, Chiba 277-8583, Japan \\ ${ }^{e}$ Institute for Theoretical Particle Physics (TTP), Karlsruhe Institute of Technology, \\ Engesserstraße 7, D-76128 Karlsruhe, Germany \\ ${ }^{f}$ Institute for Nuclear Physics (IKP), Karlsruhe Institute of Technology, \\ Hermann-von-Helmholtz-Platz 1, D-76344 Eggenstein-Leopoldshafen, Germany \\ ${ }^{g}$ KEK Theory Center, IPNS, KEK, \\ Tsukuba, Ibaraki 305-0801, Japan \\ E-mail: keisukeharigaya@berkeley.edu, ibe@icrr.u-tokyo.ac.jp, \\ teppei.kitahara@kit.edu
}

ABSTRACT: Recently the ATLAS experiment has reported $3.0 \sigma$ excess in an on- $Z$ signal region in searches for supersymmetric particles. We find that the next-to-minimal supersymmetric standard model can explain this excess by the production of gluinos which mainly decay via $\tilde{g} \rightarrow g \tilde{\chi}_{2,3}^{0} \rightarrow g Z \tilde{\chi}_{1}^{0}$ where $\tilde{\chi}_{2,3}^{0}$ and $\tilde{\chi}_{1}^{0}$ are the Higgsino and the singlinolike neutralinos, respectively. We show that the observed dark matter density is explained by the thermal relic density of the singlino-like neutralino, simultaneously. We also discuss the searches for the Higgs sector of this scenario at the Large Hadron Collider.

Keywords: Supersymmetry Phenomenology

ARXIV EPRINT: 1510.07691 


\section{Contents}

1 Introduction 1

$\begin{array}{lll}2 & \text { NMSSM } & 3\end{array}$

3 Explanation of the ATLAS on- $Z$ excess 5

4 Dark matter property $\quad 9$

5 Higgs sector searches at the LHC 11

6 Conclusion 12

\section{Introduction}

The supersymmetric (SUSY) models are attractive candidates for physics beyond the standard model (SM) because the hierarchy problem can be solved and dark matter is naturally introduced when the $R$-parity is conserved. Among various possibilities, the minimal SUSY standard model (MSSM) has been the prime candidate for the realistic supersymmetric model. A drawback of the MSSM is, however, that it contains a dimensionful parameter $\mu$, the mass term of the Higgs multiplets. This reintroduces an additional fine-tuning problem, the so-called $\mu$ problem [1]. The size of $\mu$ is required to be of the order of other soft SUSY breaking parameters for successful electroweak symmetry breaking whereas they are essentially unrelated with each other.

The simplest solution of the $\mu$ problem is to introduce an additional gauge-singlet superfield $\hat{S}[2]$ whose vacuum expectation value (VEV) is controlled by soft SUSY breaking parameters. By making an effective $\mu$ term generated by the VEV of $\hat{S}$, the size of $\mu$ is naturally interrelated to the size of the soft SUSY breaking parameters. The next-tominimal SUSY standard model (NMSSM) is one of the simplest singlet extensions of the MSSM where a discrete $\mathbb{Z}_{3}$ symmetry is imposed [2-4].

Recently, the ATLAS experiment has reported excess events in the SUSY particle searches with dileptons, jets and missing transverse energy $\left(E_{\mathrm{T}}^{\mathrm{miss}}\right)$ in data of $20.3 \mathrm{fb}^{-1}$ at $\sqrt{s}=8 \mathrm{TeV}$ [5]. They have observed 29 (16 for ee and 13 for $\mu \mu$ ) same-flavour oppositesign dilepton pairs whose invariant masses are in the $Z$ boson mass window, $81 \mathrm{GeV}<$ $m_{\ell \ell}<101 \mathrm{GeV}$ ("on- $Z$ " signal region). The expected number of SM background events is $10.6 \pm 3.2$ pairs. The observed event number corresponds to excess of $3.0 \sigma$ local significance (3.0 $\sigma$ for $e e$ and $1.7 \sigma$ for $\mu \mu$, separately). In this paper, we call this excess "ATLAS on$Z$ excess". This excess seems to imply an existence of a gluino whose mass is lighter than $1.2 \mathrm{TeV}$ or squarks lighter than $1.4 \mathrm{TeV}$ [6]. The caveat is, though, that the CMS experiment has also analyzed the dileptons + jets $+E_{\mathrm{T}}^{\text {miss }}$ final state using $\sqrt{s}=8 \mathrm{TeV}$ data 
in which the kinematical cut is different from the ATLAS one, and a significant excess has not been observed in the on- $Z$ signal region [7].

After the report, many scenarios in the MSSM as well as in the NMSSM have been proposed to explain the ATLAS on- $Z$ excess without conflicting with constraints from various SUSY searches including the CMS on- $Z$ result [6, 8-20]. To have on-shell $Z$ bosons in final states while escaping other SUSY search constraints, scenarios with a gravitino as the lightest SUSY particle (LSP) seems to be one of the simplest possibilities. The lightest neutralino decays into a pair of a $Z$ boson and a gravitino, while the decay of colored SUSY particles into the gravitino with a large $E_{\mathrm{T}}^{\mathrm{miss}}$ are suppressed. Unfortunately, however, the simplified general gauge mediation model with the gravitino LSP cannot explain the ATLAS on- $Z$ excess $[9,10]$, where the produced $Z$ bosons are rather hard due to the lightness of the gravitino and are caught in the mesh of the SUSY searches with multi jets $+E_{\mathrm{T}}^{\mathrm{miss}}$.

In ref. [16], it has been shown that this problem can be evaded by introducing a nonMSSM massive particle, a goldstini $\tilde{G}^{\prime}$, into which the lightest neutralino mainly decays. Due to the massiveness of the goldstini, the $Z$ bosons are emitted softly, and hence, the constraints from 0 lepton + multi jets $+E_{\mathrm{T}}^{\text {miss }}$ searches become weaker. In this scenario, similar to the gravitino LSP scenario, the couplings between the goldstini and the MSSM particles are suppressed. Besides, the sfermion masses are assumed to be rather larger than the gaugino masses and the lightest neutralino is assumed to be the Higgsino-like. With this setup, the ATLAS on- $Z$ excess is successfully explained by the gluino production via a decay chain, $\tilde{g} \rightarrow g \tilde{\chi}_{1,2}^{0} \rightarrow g Z \tilde{G}^{\prime}$, where $\tilde{\chi}_{1,2}^{0}$ are the Higgsino-like neutralinos.

The above goldstini nature is also realized in the NMSSM with a singlino-like neutralino LSP, where the singlino is the fermionic component of the additional singlet superfield $\hat{S}$. Actually, some literature investigated this possibility with decay chains of $\tilde{g} \rightarrow q \bar{q} \tilde{\chi}_{2}^{0} \rightarrow$ $q \bar{q} Z \tilde{\chi}_{1}^{0}[9,12]$, and $\tilde{q} \rightarrow q \tilde{\chi}_{2}^{0} \rightarrow q Z \tilde{\chi}_{1}^{0}[17,19]$, where in both cases $\tilde{\chi}_{2}^{0}$ and $\tilde{\chi}_{1}^{0}$ are the bino and singlino-like neutralinos, respectively.

In this paper, we discuss another possibility in the NMSSM and consider the gluino pair production whose decay chain is $\tilde{g} \rightarrow g \tilde{\chi}_{2,3}^{0} \rightarrow g Z \tilde{\chi}_{1}^{0}$, where $\tilde{\chi}_{2,3}^{0}$ and $\tilde{\chi}_{1}^{0}$ are the Higgsino-like and the singlino-like neutralinos, respectively (see figure 1). It should be noted that the two-body gluino decay modes $\tilde{g} \rightarrow g \tilde{\chi}_{2,3}^{0}$ at one-loop level are the dominant ones when the mass differences between the gluino and the Higgsinos are moderate and the squark masses are in the several TeV range [15, 21, 22]. Besides, as we will show, the radiative decay of the gluino reproduces the distribution of the jet multiplicity observed in the ATLAS results well.

We also investigate the properties of dark matter in the NMSSM, and find two distinct benchmark parameter sets. Eventually, we show that the ATLAS on- $Z$ excess and the observed relic abundance of dark matter can be simultaneously explained in the NMSSM, without conflicting with other experimental constraints, including the CMS on- $Z$ result.

We organize the rest of this paper as follows. In section 2, we briefly review the mass matrices of the Higgs-sector and neutralinos in the NMSSM. Next in section 3, we give an explanation of the ATLAS on- $Z$ excess using the NMSSM with the singlino-like neutralino LSP. In section 4 , the property of the dark matter around our benchmark points 


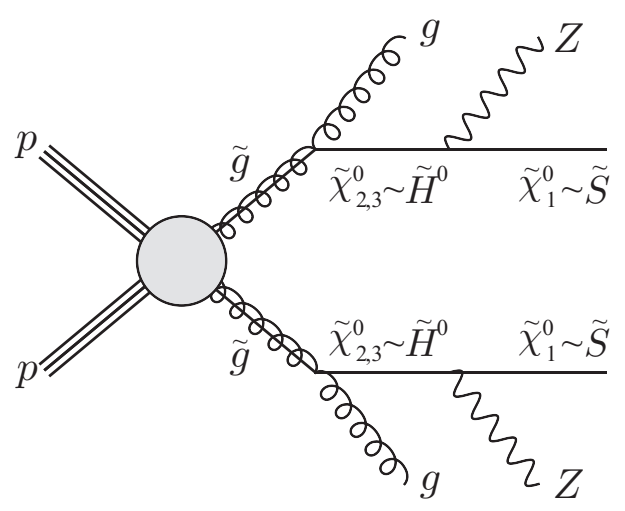

Figure 1. A typical diagram of the gluino decay in the scenario which we considered. See section 3 for details.

is discussed. In section 5, we discuss the searches for the Higgs sector at the LHC. The final section is devoted to conclusion.

\section{NMSSM}

In this paper, we investigate a possible explanation of the ATLAS on- $Z$ excess in the NMSSM. Motivated by the study about the goldstini [16], we consider the case of the singlino-like neutralino LSP.

In this section, we quickly review the mass spectrum of the NMSSM by paying particular attention to the dependence on parameters. The detail of the NMSSM is given in ref. [23]. The superpotential and the scalar potential of the Higgs-sector in the NMSSM are given as

$$
\begin{aligned}
W= & \lambda \hat{S} \hat{H}_{2} \hat{H}_{1}+\frac{\kappa}{3} \hat{S}^{3}, \\
V= & \left(|\lambda S|^{2}+m_{1}^{2}\right)\left|H_{1}\right|^{2}+\left(|\lambda S|^{2}+m_{2}^{2}\right)\left|H_{2}\right|^{2} \\
& +\frac{g^{2}}{2}\left|\left(H_{1}^{\dagger} H_{2}\right)\right|^{2}+\frac{g^{2}+g^{\prime 2}}{8}\left(\left|H_{1}\right|^{2}-\left|H_{2}\right|^{2}\right)^{2} \\
& +\left|\lambda H_{2} H_{1}+\kappa S^{2}\right|^{2}+m_{s}^{2}|S|^{2}+\left(\lambda A_{\lambda} S H_{2} H_{1}+\frac{\kappa}{3} A_{\kappa} S^{3}+\text { H.c. }\right),
\end{aligned}
$$

where fields with hats are superfields, and $\hat{S}, \hat{H}_{1,2}$ are the singlet and the down-type and up-type Higgs doublets, respectively. $\kappa$ and $\lambda$ are coupling constants in the superpotential, and $g$ and $g^{\prime}$ are gauge coupling constants of the $\mathrm{SU}(2)_{L}$ and $\mathrm{U}(1)_{Y}$ gauge interactions. Due to the discrete $\mathbb{Z}_{3}$ symmetry under which $\hat{S}$ as well as $\hat{H}_{1,2}$ rotate with unit charges, the superpotential does not have dimensionful parameters. The dimensionful parameters in the scalar potential are the soft SUSY breaking parameters, i.e., $m_{1,2, s}^{2}$ and $A_{\lambda, \kappa}$.

For simplicity, we assume all parameters to be real in this paper. When the electroweak symmetry is broken, three neutral scalar bosons obtain VEVs and they are expanded around their VEVs as follows,

$$
H_{1}^{0}=v_{1}+\frac{H_{1 R}+i H_{1 I}}{\sqrt{2}}, \quad H_{2}^{0}=v_{2}+\frac{H_{2 R}+i H_{2 I}}{\sqrt{2}}, \quad S=v_{s}+\frac{S_{R}+i S_{I}}{\sqrt{2}},
$$


where $v^{2}=v_{1}^{2}+v_{2}^{2} \simeq(174.1 \mathrm{GeV})^{2}$ and we use $\tan \beta \equiv v_{2} / v_{1}$ in the following. Then the effective $\mu$ term is generated,

$$
\mu_{\mathrm{eff}}=\lambda v_{s}
$$

Since the singlet VEV $v_{s}$ is the same scale as the SUSY breaking dimensionful terms, the $\mu$ problem is solved.

In the NMSSM, the singlino is the one of the neutralino components. The neutralino mass matrix for the basis $\psi^{0}=\left(\tilde{B}, \tilde{W}^{0}, \tilde{H}_{1}^{0}, \tilde{H}_{2}^{0}, \tilde{S}\right)$ is given as

$$
\mathcal{L}=-\frac{1}{2}\left(\psi^{0}\right)^{T} M_{\tilde{N}} \psi^{0}+\text { H.c. }
$$

with

$$
M_{\tilde{N}}=\left(\begin{array}{ccccc}
M_{1} & 0 & -\frac{g^{\prime} v_{1}}{\sqrt{2}} & \frac{g^{\prime} v_{2}}{\sqrt{2}} & 0 \\
0 & M_{2} & \frac{g v_{1}}{\sqrt{2}} & -\frac{g v_{2}}{\sqrt{2}} & 0 \\
-\frac{g^{\prime} v_{1}}{\sqrt{2}} & \frac{g v_{1}}{\sqrt{2}} & 0 & -\mu_{\mathrm{eff}} & -\lambda v_{2} \\
\frac{g^{\prime} v_{2}}{\sqrt{2}} & -\frac{g v_{2}}{\sqrt{2}} & -\mu_{\mathrm{eff}} & 0 & -\lambda v_{1} \\
0 & 0 & -\lambda v_{2} & -\lambda v_{1} & 2 \kappa v_{s}
\end{array}\right) .
$$

Typically, the mass of the singlino-like neutralino is given as $m_{\tilde{S}} \simeq\left|2 \kappa v_{s}\right|$.

The Higgs mass matrices for the basis $\left(H^{\prime}, h^{\prime}, S_{R}\right)$ and $\left(A^{\prime}, S_{I}, G\right)$ (defined below) are given as

$$
\begin{aligned}
V= & \frac{1}{2}\left(\begin{array}{lll}
H^{\prime} & h^{\prime} & S_{R}
\end{array}\right)\left(\begin{array}{ccc}
\tilde{\mathcal{M}}_{R 11}^{2} & \tilde{\mathcal{M}}_{R 12}^{2} & \tilde{\mathcal{M}}_{R 13}^{2} \\
\tilde{\mathcal{M}}_{R 12}^{2} & \tilde{\mathcal{M}}_{R 22}^{2} & \tilde{\mathcal{M}}_{R 23}^{2} \\
\tilde{\mathcal{M}}_{R 13}^{2} & \tilde{\mathcal{M}}_{R 23}^{2} & \tilde{\mathcal{M}}_{R 33}^{2}
\end{array}\right)\left(\begin{array}{c}
H^{\prime} \\
h^{\prime} \\
S_{R}
\end{array}\right) \\
& +\frac{1}{2}\left(\begin{array}{lll}
A^{\prime} & S_{I} & G
\end{array}\right)\left(\begin{array}{ccc}
\tilde{\mathcal{M}}_{I 11}^{2} & \tilde{\mathcal{M}}_{I 12}^{2} & 0 \\
\tilde{\mathcal{M}}_{I 12}^{2} & \tilde{\mathcal{M}}_{I 22}^{2} & 0 \\
0 & 0 & 0
\end{array}\right)\left(\begin{array}{c}
A^{\prime} \\
S_{I} \\
G
\end{array}\right) .
\end{aligned}
$$

with

$$
\begin{aligned}
\tilde{\mathcal{M}}_{R 11}^{2} & =M_{A}^{2}+M_{Z}^{2} \sin ^{2} 2 \beta+\lambda^{2} v^{2} \cos ^{2} 2 \beta, \\
\tilde{\mathcal{M}}_{R 22}^{2} & =M_{Z}^{2} \cos ^{2} 2 \beta+\lambda^{2} v^{2} \sin ^{2} 2 \beta, \\
\tilde{\mathcal{M}}_{R 33}^{2} & =\frac{\lambda A_{\lambda} v^{2}}{2 v_{s}} \sin 2 \beta+\kappa v_{s}\left(A_{\kappa}+4 \kappa v_{s}\right), \\
\tilde{\mathcal{M}}_{R 12}^{2} & =M_{Z}^{2} \sin 2 \beta \cos 2 \beta-\lambda^{2} v^{2} \sin 2 \beta \cos 2 \beta, \\
\tilde{\mathcal{M}}_{R 13}^{2} & =\lambda v\left(2 \kappa v_{s}+A_{\lambda}\right) \cos 2 \beta, \\
\tilde{\mathcal{M}}_{R 23}^{2} & =\lambda v\left(2 \lambda v_{s}-\left(2 \kappa v_{s}+A_{\lambda}\right) \sin 2 \beta\right), \\
\tilde{\mathcal{M}}_{I 11}^{2} & =M_{A}^{2}+\lambda^{2} v^{2}, \\
\tilde{\mathcal{M}}_{I 22}^{2} & =\frac{\lambda A_{\lambda} v^{2}}{2 v_{s}} \sin 2 \beta+2 \lambda \kappa v^{2} \sin 2 \beta-3 \kappa A_{\kappa} v_{s}, \\
\tilde{\mathcal{M}}_{I 12}^{2} & =\lambda v\left(-2 \kappa v_{s}+A_{\lambda}\right),
\end{aligned}
$$


where

$$
M_{A}^{2} \equiv \frac{2 \mu_{\mathrm{eff}}\left(A_{\lambda}+\kappa v_{s}\right)}{\sin 2 \beta}-\lambda^{2} v^{2} .
$$

Here, the convenient basis $\left(H^{\prime}, h^{\prime}, S_{R}\right)$ and $\left(A^{\prime}, S_{I}, G\right)$ are defined by

$$
\begin{aligned}
&\left(\begin{array}{c}
H_{1 R} \\
H_{2 R} \\
S_{R}
\end{array}\right) \equiv\left(\begin{array}{ccc}
\sin \beta & \cos \beta & 0 \\
-\cos \beta & \sin \beta & 0 \\
0 & 0 & 1
\end{array}\right)\left(\begin{array}{c}
H^{\prime} \\
h^{\prime} \\
S_{R}
\end{array}\right), \\
&\left(\begin{array}{c}
H_{1 I} \\
H_{2 I} \\
S_{I}
\end{array}\right) \equiv\left(\begin{array}{ccc}
\sin \beta & 0 & -\cos \beta \\
\cos \beta & 0 & \sin \beta \\
0 & 1 & 0
\end{array}\right)\left(\begin{array}{c}
A^{\prime} \\
S_{I} \\
G
\end{array}\right) .
\end{aligned}
$$

One of the NMSSM specific $A$ terms $A_{\lambda}$ plays two important roles in determining the properties of the singlet particles and the Higgs mass spectrum. First, the mass scale of the heavy Higgs in eq. (2.17) can be controlled by $A_{\lambda}$. Second, a singlet-doublet mixing for the SM-like Higgs boson can also be controlled by $A_{\lambda}$. Even if the mass of the singlet-like Higgs boson is heavy, the effect of the singlet-doublet mixing does not decouple. When the lightest $\mathrm{CP}$ even Higgs boson is the SM-like one and $M_{A}$ is very large, the contribution of an off-diagonal mass term $\tilde{\mathcal{M}}_{R 23}^{2}$ (eq. (2.13)) to the mass of the SM-like Higgs boson is roughly estimated as follows, ${ }^{1}$

$$
\Delta m_{h_{1}}^{2} \sim-\frac{\left(\tilde{\mathcal{M}}_{R 23}^{2}\right)^{2}}{\tilde{\mathcal{M}}_{R 33}^{2}} \sim-\mathcal{O}\left(v^{2}\right),
$$

which leads to a too light SM-like Higgs boson mass. This undesirable negative contribution via the singlet-doublet mixing can be avoided if $\lambda \ll 1$ or when the following condition is satisfied,

$$
A_{\lambda}=\frac{2 \lambda v_{s}}{\sin 2 \beta}-2 \kappa v_{s} \sim \frac{2 m_{\tilde{H}}}{\sin 2 \beta}-m_{\tilde{S}}
$$

These conditions also suppress the off-diagonal mass term $\tilde{\mathcal{M}}_{R 23}^{2}$.

\section{Explanation of the ATLAS on- $Z$ excess}

In this section, we give an explanation of the ATLAS $Z+$ jets $+E_{\mathrm{T}}^{\text {miss }}$ excess in the NMSSM. In order to explain the signals of the ATLAS on- $Z$ excess by the gluino pair production, we consider the following mass spectrum,

$$
\begin{aligned}
m_{\tilde{g}} & \lesssim 1 \mathrm{TeV}, \\
m_{\text {Higgsino,NLSP }} & \gtrsim m_{\tilde{g}}-300 \mathrm{GeV}, \\
m_{\text {singlino,LSP }} & \simeq m_{\text {Higgsino,NLSP }}-100 \mathrm{GeV} .
\end{aligned}
$$

The reason to choose the above mass spectrum is described below.

\footnotetext{
${ }^{1}$ On the other hand, the contribution to the singlet component of the SM-like Higgs mass eigenstate is decoupled in proportion to $\tilde{\mathcal{M}}_{R 23}^{2} / \tilde{\mathcal{M}}_{R 33}^{2}$.
} 
In order to explain the ATLAS on- $Z$ excess by the gluino production, the following items are required.

- The gluino mass is required to be lighter than about $1.2 \mathrm{TeV}$ for a large enough production cross section to explain the number of the excess [6].

- In the dominant gluino decay chain, at least one $Z$ boson emission like $\tilde{g} \rightarrow X+$ NLSP $\rightarrow X+Z+$ LSP is required, where $X$ denotes SM colored particles.

- Since $Z$ bosons dominantly decay hadronically, multi jets $+E_{\mathrm{T}}^{\mathrm{miss}}$ searches put severe constraints. In order to ameliorate these constraints, the $Z$ boson is required to be emitted rather softly. In addition, the decay of the NLSP into the Higgs boson + LSP is required to be suppressed so that the $Z$ boson production is enhanced. This requirement also helps to evade the multi-jets constraints caused by the Higgs boson decay.

- In order to shift the jet multiplicity distribution to smaller values as is favored by the ATLAS result, the loop-induced gluino two-body decay is required to become dominant channel. This requirement is also advantageous to evade the multi-jets constraints.

To satisfy the above conditions, we consider the following mass spectrum in the NMSSM. First, in order to enhance the soft $Z$ boson production, we assume a slight degeneracy between the NLSP and the LSP; $m_{Z}<m_{\mathrm{NLSP}}-m_{\mathrm{LSP}}<m_{h}$.

Next, in order for the gluino two-body decay to be a dominant channel, we assume that the gluino is lighter than the squarks, the bino, and the wino, while it is heavier than the Higgsinos and the singlino. ${ }^{2}$ Besides, to suppress the decay of the gluino into $t \bar{t}(t \bar{b}, b \bar{t})+$ Higgsino kinematically, we assume a slight degeneracy between the gluino and the Higgsino; $m_{\tilde{g}}-m_{\text {Higgsino }} \lesssim 300 \mathrm{GeV}$. Finally, to suppress the decay of the gluino into $b \bar{b}+$ Higgsino, we take the squark masses to be of order of $10 \mathrm{TeV}$. Note that the loopinduced gluino two-body decay into the gluon + Higgsino is relatively enhanced by a factor $\left(\ln \left(m_{\tilde{t}} / m_{t}\right)\right)^{2}[15,21,22]$.

With the above mass spectrum, the $Z$ boson is produced by the decay of the NLSP Higgsino-like neutralino into the LSP singlino-like neutralino. In terms of the model parameters, the Higgsino mass is typically given by $\left|\lambda v_{s}\right|$ and the singlino mass is given by $\left|2 \kappa v_{s}\right|$. Hence, $\lambda \sim 2 \kappa$ to achieve the above mass spectrum.

Altogether, we consider the mass spectrum in eq. (3.1), in which the typical decay chain of the gluino is $\tilde{g} \rightarrow g \tilde{\chi}_{2,3}^{0} \rightarrow g Z \tilde{\chi}_{1}^{0}$, where $\tilde{\chi}_{2,3}^{0}$ and $\tilde{\chi}_{1}^{0}$ are the Higgsino and the singlino-like neutralinos, respectively. The diagram is drawn in figure 1 . Note that due to small couplings between the singlino and the colored sector, the direct decay of the gluino into the singlino-like neutralino is suppressed. This is the advantage of the singlino-like

\footnotetext{
${ }^{2}$ This spectrum requires non-universal gaugino masses. We note that the doublet-triplet splitting of the Higgs is naturally achieved in grand unified theories with product gauge groups [24] (see ref. [25] and references therein for related discussions), which in general predict non-universal gaugino masses.
} 
LSP compared with the bino LSP scenario where the gluino decay mode into the bino LSP with jets can be comparable to the mode into the Higgsino NLSP depending on $\tan \beta$.

Keeping above arguments in mind, we find two distinct valid parameter regions where the ATLAS on- $Z$ excess can be explained. One is a region with a small $\lambda$ and the other is the one with a large $\lambda$. As we will discuss in the next section, these two regions have different dark matter properties. It should be noted that the NMSSM in the small $\lambda$ region is almost the same with the MSSM plus an additional gauge singlet fermion whose couplings to the MSSM sector are suppressed as in the simplified goldstini model [16]. In this region, the analyses of ref. [16] would be applied. In table 1, we show the two benchmark parameter sets in the two distinct parameter regions which exemplify the small $\lambda$ and the large $\lambda$ regions, respectively. In our analysis, we use the spectrum calculator NMSSMTools $4.7 .0[26,27]$ and also use the decay width calculator NMSDECAY [28]. In the table, we also show the properties of the dark matter which are calculated using MicrOMEGAs4.1.8 [29, 30] (see next section). Note that we take $M_{1}=M_{2}=1.5 \mathrm{TeV}$ in both benchmark points, and the bino and winos are decoupled enough.

At the small $\lambda$ benchmark point, the mass of the SM-like Higgs boson is given by the radiative correction from the stop loop diagrams, which requires a large $\tan \beta$. This large $\tan \beta$ leads to a large decay width of $\tilde{g} \rightarrow b \bar{b}(t \bar{b}, b \bar{t})+$ Higgsino via an enhancement of the bottom Yukawa. Even for a rather large $\tan \beta$, however, we have confirmed that the dominant decay channel of the gluino is $\tilde{g} \rightarrow g+$ Higgsino (see table 1).

At the large $\lambda$ benchmark point, the mass of the SM-like Higgs boson is given by both the radiative correction and an additional $F$-term contribution which requires a small $\tan \beta$ (see eq. (2.9)). Such a large $\lambda$ also brings the undesirable negative contribution to the lightest CP-even Higgs mass in eq. (2.20). To avoid this problem, we choose $A_{\lambda}$ that suppresses this negative contribution according to eq. (2.21). ${ }^{3}$ Note that when one takes $A_{\lambda}$ to be $\mathcal{O}(1) \mathrm{TeV}$, this equation also suggests the small $\tan \beta$. The large $\lambda$ also leads to a certain singlino-Higgsino mixing, so that the decay branch $\tilde{g} \rightarrow g+$ singlino-like neutralino exists. Even with such contributions, we have again confirmed that the dominant decay channel is $\tilde{g} \rightarrow g+$ Higgsino (see table 1).

To investigate the number of the SUSY events in the ATLAS on- $Z$ search and to check the experimental constraints from the other SUSY searches at the ATLAS and CMS collaborations, we use CheckMATE 1.2.1 [12, 31] which incorporates DELPHES 3 [32] and FastJet $[33,34]$ internally. Signal events are generated by MadGraph5 v2.2.3 [35, 36] connected to Pythia 6.4 [37] where the MLM matching scheme is used with a matching scale at $150 \mathrm{GeV}$ [38]. The parton distribution functions are CTEQ6L1 [39]. We use the gluino production cross sections at the next-to-leading-logarithmic accuracy given in ref. [40] with NLL-fast [41-45].

As a result, we find that the SUSY events can explain the ATLAS on- $Z$ excess within $1 \sigma$ without conflicting with any LHC constraints at 95\% CL, including the CMS on- $Z$ result for both benchmark points. In addition, the lightest CP-even Higgs boson mass is

\footnotetext{
${ }^{3}$ The singlet component of the lightest CP-even Higgs boson is suppressed by a large singlet scalar mass. Then the Higgs couplings are almost equivalent to the ones in the SM even if we do not choose such a $A_{\lambda}$.
} 


\begin{tabular}{|c|c|c|}
\hline & small $\lambda$ & large $\lambda$ \\
\hline$\lambda$ & 0.080 & 0.435 \\
\hline$\kappa$ & 0.033 & 0.185 \\
\hline $\tan \beta$ & 30 & 3 \\
\hline$A_{\lambda}[\mathrm{GeV}]$ & -146 & 1500 \\
\hline$A_{\kappa}[\mathrm{GeV}]$ & -50 & -200 \\
\hline$\mu_{\mathrm{eff}}[\mathrm{GeV}]$ & 620 & 600 \\
\hline$m_{\tilde{q}}[\mathrm{TeV}]$ & 10 & 10 \\
\hline$A_{q}[\mathrm{TeV}]$ & 6 & 0 \\
\hline$m_{\tilde{g}}[\mathrm{GeV}]$ & 900 & 925 \\
\hline$m_{\tilde{\chi}_{3}^{0}}[\mathrm{GeV}]$ & 641 & 625 \\
\hline$m_{\tilde{\chi}_{2}^{0}}[\mathrm{GeV}]$ & 636 & 624 \\
\hline$m_{\tilde{\chi}_{1}^{0}}[\mathrm{GeV}]$ & 527 & 519 \\
\hline$m_{\tilde{\chi}_{1}^{ \pm}}[\mathrm{GeV}]$ & 637 & 615 \\
\hline$m_{h_{1}}[\mathrm{GeV}]$ & 125 & 125 \\
\hline$m_{h_{2}}[\mathrm{GeV}]$ & 500 & 453 \\
\hline$m_{h_{3}}[\mathrm{GeV}]$ & 1061 & 1831 \\
\hline$m_{a_{1}}[\mathrm{GeV}]$ & 195 & 439 \\
\hline$m_{a_{2}}[\mathrm{GeV}]$ & 1061 & 1829 \\
\hline$m_{H^{+}}[\mathrm{GeV}]$ & 1056 & 1822 \\
\hline $\operatorname{Br}\left(\tilde{g} \rightarrow g \tilde{\chi}_{2,3}^{0}\right)$ & 0.79 & 0.70 \\
\hline $\operatorname{Br}\left(\tilde{g} \rightarrow g \tilde{\chi}_{1}^{0}\right)$ & 0.008 & 0.12 \\
\hline $\operatorname{Br}(\tilde{g} \rightarrow$ others $)$ & $0.20\left(b \bar{b} \tilde{\chi}_{2,3}^{0}\right)$ & $0.18\left(t b \tilde{\chi}_{1}^{ \pm}\right)$ \\
\hline $\operatorname{Br}\left(\tilde{\chi}_{2,3}^{0} \rightarrow Z \tilde{\chi}_{1}^{0}\right)$ & 1.00 & 1.00 \\
\hline SUSY events in ATLAS on- $Z$ & 14 & 14 \\
\hline$\Omega_{\tilde{\chi}} h^{2}$ & 0.118 & 0.121 \\
\hline$\sigma_{\mathrm{SI}}\left[\mathrm{cm}^{2}\right]$ & $4.0 \times 10^{-47}$ & $2.8 \times 10^{-45}$ \\
\hline Higgs coupling $\kappa_{V}$ & 0.997 & 0.9997 \\
\hline Higgs coupling $\kappa_{b}$ & 1.02 & 1.02 \\
\hline
\end{tabular}

Table 1. Two benchmark parameter sets. In the line of $\operatorname{Br}(\tilde{g} \rightarrow$ others $)$, parentheses represent the dominant decay channel. In both benchmark points, the lightest neutralino $\tilde{\chi}_{1}^{0}$, the second lightest CP-even Higgs $h_{2}$, and the lightest CP-odd Higgs $a_{1}$ are the mass eigenstates dominated by singlet contributions.

$125 \mathrm{GeV}$ and the relic abundance of the singlino-like neutralino is also consistent with the observed values within $2 \sigma$ [46].

Let us comment on the uncertainty in the calculation of the Higgs boson mass. In NMSSMTools 4.7.0, the Higgs boson masses are calculated at full one-loop plus two-loop $\mathcal{O}\left(\alpha_{t} \alpha_{s}+\alpha_{b} \alpha_{s}\right)$ level [47]. When the squark mass is about $10 \mathrm{TeV}$, a theoretical uncertainty of the SM-like Higgs boson mass from higher order corrections by the MSSM particles is about $5 \mathrm{GeV}$. Including the higher order corrections within the effective field theory approach, this uncertainty can be reduced to around $1 \mathrm{GeV}$ with the similar central value 
in the case of the MSSM [48]. Thus, we simply use the result of NMSSMTools 4.7.0. Note that FeynHiggs [49] yields a larger Higgs mass by about $5 \mathrm{GeV}$. If this is the case, the required squark mass should be smaller by a factor of a few and the branching ratio of the radiative decay of the gluino becomes smaller. In the case of the large $\lambda$, we may change $\tan \beta$ as well as $\lambda$ and obtain the similar LHC signal.

Before closing this section, let us comment on the distributions of the jet multiplicity, $E_{\mathrm{T}}^{\mathrm{miss}}$ and $H_{T}$ in the on- $Z$ signal region. In figure 2, we show the distributions of the jet multiplicity, $E_{\mathrm{T}}^{\mathrm{miss}}$ and $H_{T}$ for the small $\lambda$ and large $\lambda$ benchmark points, respectively. Note that we combine the $e e$ and $\mu \mu$ channels of the ATLAS results, although the efficiencies of the channels are different from each other. As shown in ref. [5], the jet multiplicity of the observed data is typically $2-5$ jets while the multiplicity larger than 6 is disfavored. For both benchmark points, the jet multiplicity of the NMSSM contributions is peaked at around $4-5$, which originates from two gluon jets in two gluino decays, two quarks from a $Z$-boson decay in one of the gluino decay chain, and an occasionally radiated jet. The predicted distributions of the jet multiplicity fit the ATLAS data very well, which confirms the advantage of the dominance of the gluino two body decay [16]. It can be seen that the distributions of $E_{\mathrm{T}}^{\mathrm{miss}}$ and $H_{T}$ are also consistent with the data.

\section{Dark matter property}

In this section, we discuss the properties of dark matter in the small and large $\lambda$ regions as exemplified in the above two benchmark points (see table 1). In the small $\lambda$ region, the lightest neutralino is almost singlino-like. In most cases, such a singlino-like LSP results in overabundant dark matter in the universe because its annihilation cross section is too small. The overabundance is, however, avoided when the heavy Higgs masses are about twice of the mass of the singlino dark matter. Such a mass spectrum can be achieved by tuning the parameter $A_{\lambda}[50]$. With this tuning, the singlino-like neutralino annihilates resonantly via the $s$-channel heavy Higgs boson exchange, which significantly enhances the annihilation cross section. ${ }^{4}$ In this way, the observed dark matter density is explained by the singlino-like neutralino at the small $\lambda$ benchmark point in spite of the weakness of couplings. It should be noted that due to a suppressed coupling between the singlino and the SM-like Higgs boson, the spin-independent dark matter-nucleon elastic scattering cross section is much lower than the reaches in currently proposed direct dark mater searches.

In the large $\lambda$ region, on the other hand, there is a certain singlino-Higgsino mixing. The Higgsino components in the LSP can enhance the annihilation cross section, so that the tuning of the heavy Higgs mass is not required. In addition, sizable $\kappa$ can also enhance the annihilation cross section. It is because that contributions of diagrams of $s$-cannel $a_{s}$ boson exchange into fermions [53] and $t$-channel $\tilde{\chi}_{1}^{0}$ exchange into $h_{s}+a_{s}$ [50] become efficient when $\kappa$ is sizable, where both $a_{s}$ and $h_{s}$ are the singlet-like scalar bosons. In fact, at the large $\lambda$ benchmark point, the contribution of a process $\tilde{\chi}_{1}^{0} \tilde{\chi}_{1}^{0} \rightarrow t \bar{t}$ to the annihilation cross section is $79 \%$, and $\tilde{\chi}_{1}^{0} \tilde{\chi}_{1}^{0} \rightarrow h_{2} a_{1}$ is $15 \%$, where a dominant decay channel of $a_{1}$ is $t \bar{t}$.

\footnotetext{
${ }^{4}$ The other possibility is a resonant annihilation via the SM Higgs or $Z$ boson [50-52]. However, due to a large mass difference between the NLSP and singlino, the emitted $Z$ boson becomes energetic.
} 

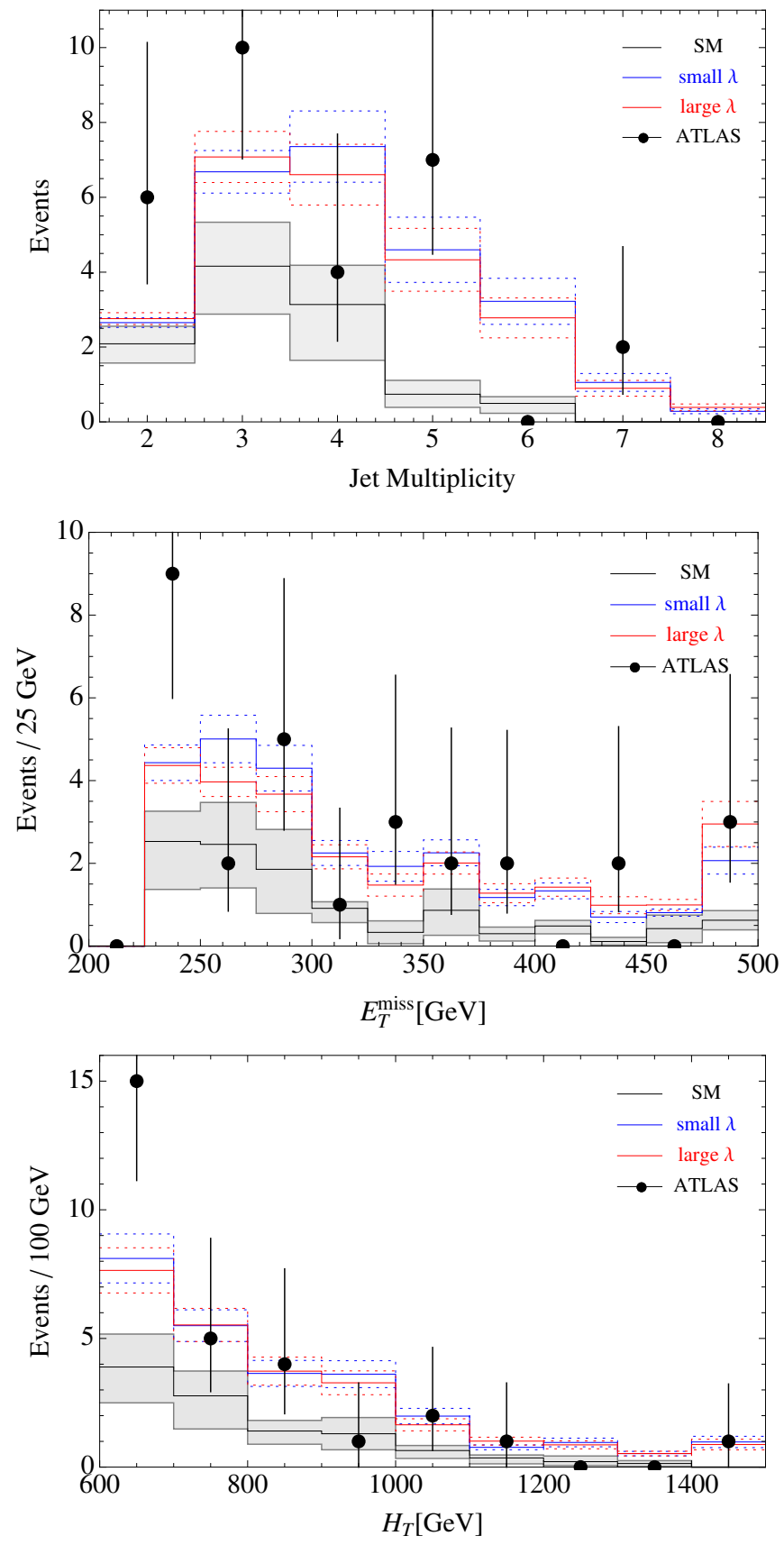

Figure 2. The distributions of the jet multiplicity (top), $E_{\mathrm{T}}^{\text {miss }}$ (middle) and $H_{T}$ (bottom) in the ATLAS on- $Z$ signal region. The ATLAS data points are shown by black points with combined errors. The black line shows the Standard Model contributions whose errors are shown by gray shade. The expected distributions for the two benchmark points are shown by the blue (small $\lambda$ ) and the red (large $\lambda$ ) lines. The dotted lines represent the error of the NMSSM contributions which is dominated by the uncertainty of the gluino production cross section of about $30 \%$. We ignore the uncertainty of the estimation of the efficiency in CheckMATE 1.2.1. 
The singlino-Higgsino mixing also contributes to the spin-independent dark matternucleon elastic scattering cross section at the large $\lambda$ benchmark point. We found that the typical scattering cross section around the benchmark point is a little smaller than a current experimental bound by LUX [54], and proposed future experiments for the direct dark matter search can probe around this benchmark point [55-58].

\section{Higgs sector searches at the LHC}

In this section, we discuss the searches for the Higgs sector at the LHC. First, we consider the heavy doublet-like Higgses $h_{3}$ and $a_{2}$. Successful parameter points in the small $\lambda$ region always predict that the heavy doublet-like Higgses have masses around $1 \mathrm{TeV}$ so that the singlino-like neutralino annihilates resonantly via the s-channel heavy Higgses exchanges. Interestingly, the $14 \mathrm{TeV}$ LHC with a luminosity of $300 \mathrm{fb}^{-1}$ can probe such a charged Higgs directly by the channel of $p p \rightarrow t b H^{+} \rightarrow t b t b$ due to a $\tan \beta$ enhancement of the bottom Yukawa [59]. ${ }^{5}$ In the large $\lambda$ region, although the production cross section of the heavy doublet-like Higgses is small, a high-luminosity LHC can probe them through the top Yukawa via the same channel [59].

Next, we consider the singlet-like scalars $h_{2}$ and $a_{1}$. The masses of the singlet-like scalars tend to be within the $\mathrm{TeV}$ range in our scenario. This is because typical masses of the singlet scalars are controlled by $\kappa v_{s}$, which also determines the singlino mass and is taken as about $500 \mathrm{GeV}$ in our scenario.

The singlet-like CP-even Higgs boson $h_{2}$ mainly decays into $W W$ and $Z Z$ due to an enhancement of the longitudinally polarized gauge bosons when the decay width into double Higgs, $h_{1} h_{1}$, is suppressed. In our benchmark points, we find that the gluongluon (vector boson) fusion cross sections of $p p \rightarrow h_{2}(j j) \rightarrow W W(j j)$ are $8 \mathrm{fb}(1 \mathrm{fb})$ at the small $\lambda$ benchmark point, and $0.2-20 \mathrm{fb}(0.2-4 \mathrm{fb})$ at the large $\lambda$ benchmark point for $\sqrt{s}=14 \mathrm{TeV}$, where we vary $A_{\lambda}$ in the range of $1500-1100 \mathrm{GeV}$ in the large $\lambda$ benchmark point. Within this range of $A_{\lambda}$, the reduction of the SM-like Higgs boson mass (see eq. (2.20)) can be compensated by taking a large trilinear coupling between the up-type Higgs doublet and stops. For a smaller $A_{\lambda}, h_{2}$ contains more doublet components and hence the production cross section is larger. The current experimental upper bounds for the gluon-gluon (vector boson) fusion cross sections are $200(100) \mathrm{fb}$ for $m_{h_{2}}=500 \mathrm{GeV}$ at $\sqrt{s}=8 \mathrm{TeV}[62]$.

On the other hand, the singlet-like CP-odd Higgs boson $a_{1}$ mainly decays into $t \bar{t}$. The cross section of $p p \rightarrow a_{1} \rightarrow t \bar{t}$ is $1 \mathrm{fb}$ at $\sqrt{s}=14 \mathrm{TeV}$ at the large $\lambda$ benchmark point. ${ }^{6}$ The current upper bound is $2 \mathrm{pb}$ for $m_{a_{1}}=500 \mathrm{GeV}$ at $\sqrt{s}=8 \mathrm{TeV}$ [63]. Therefore, the direct search for such the singlet-like Higgses would be challenging. It is recently shown that the precision measurement of the double Higgs production can probe the singletlike Higgs bosons through the interference effect between a resonance production of the

\footnotetext{
${ }^{5}$ In the small $\lambda$ region, another promising channel for the heavy doublet-like Higgs searches is $p p \rightarrow$ $h_{3} / a_{2} \rightarrow \tau \tau[60,61]$.

${ }^{6}$ In the small $\lambda$ benchmark point, $a_{1} \rightarrow t \bar{t}$ is forbidden by the kinematics, and mainly decays into $b \bar{b}$. However, $p p \rightarrow a_{1} \rightarrow b \bar{b}$ cannot be probed due to enormous background.
} 
singlet-like Higgs and SM processes [64-68]. This resonance signal, however, would become narrower than results in the literature due to the small singlet-doublet mixing in our benchmark points.

\section{Conclusion}

In this paper, we have studied a possible explanation of the ATLAS on- $Z$ excess in the NMSSM by the gluino production via typical decay chains, $\tilde{g} \rightarrow g \tilde{\chi}_{2,3}^{0} \rightarrow g Z \tilde{\chi}_{1}^{0}$, with $\tilde{\chi}_{2,3}^{0}$ and $\tilde{\chi}_{1}^{0}$ being the Higgsino and the singlino-like neutralinos, respectively. We found two distinct benchmark parameter sets. At the benchmark points, the observed dark matter density is also explained by the thermal relic abundance of the singlino-like neutralino. In addition, it is found that the expected distributions of the jet multiplicity, $E_{\mathrm{T}}^{\mathrm{miss}}$ and $H_{T}$ for our benchmark points are consistent with the ATLAS data.

In the small $\lambda$ region, we find that the $14 \mathrm{TeV}$ LHC with a luminosity of $300 \mathrm{fb}^{-1}$ can probe the $1 \mathrm{TeV}$ charged Higgs directly by the channel of $p p \rightarrow t b H^{+} \rightarrow t b t b$. On the other hand, in the large $\lambda$ region, we find that proposed future experiments for the direct dark matter search can probe our scenario at around the benchmark point.

\section{Acknowledgments}

The authors would like to thank Satoshi Shirai for useful discussions. This work is supported by Grants-in-Aid for Scientific Research from the Ministry of Education, Culture, Sports, Science, and Technology (MEXT) KAKENHI, Japan, No. 24740151 and No. 15 H05889 (M. I.), and No. 25105011 (M. I. and T. K.); Grant-in-Aid No. 26287039 (M. I.) from the Japan Society for the Promotion of Science (JSPS) KAKENHI; and by the World Premier International Research Center Initiative (WPI), MEXT, Japan (M. I.). K. H. was supported in part by a JSPS Research Fellowship for Young Scientists.

Open Access. This article is distributed under the terms of the Creative Commons Attribution License (CC-BY 4.0), which permits any use, distribution and reproduction in any medium, provided the original author(s) and source are credited.

\section{References}

[1] J.E. Kim and H.P. Nilles, The $\mu$-Problem and the Strong CP-Problem, Phys. Lett. B 138 (1984) 150 [INSPIRE].

[2] P. Fayet, Supergauge invariant extension of the Higgs mechanism and a model for the electron and its neutrino, Nucl. Phys. B 90 (1975) 104 [INSPIRE].

[3] H.P. Nilles, M. Srednicki and D. Wyler, Weak Interaction Breakdown Induced by Supergravity, Phys. Lett. B 120 (1983) 346 [INSPIRE].

[4] J.M. Frere, D.R.T. Jones and S. Raby, Fermion Masses and Induction of the Weak Scale by Supergravity, Nucl. Phys. B 222 (1983) 11 [INSPIRE]. 
[5] ATLAS collaboration, Search for supersymmetry in events containing a same-flavour opposite-sign dilepton pair, jets and large missing transverse momentum in $\sqrt{s}=8 \mathrm{TeV} p p$ collisions with the ATLAS detector, Eur. Phys. J. C 75 (2015) 318 [arXiv:1503.03290] [INSPIRE].

[6] G. Barenboim, J. Bernabeu, V.A. Mitsou, E. Romero, E. Torro and O. Vives, METing SUSY on the $Z$ peak, arXiv:1503.04184 [inSPIRE].

[7] CMS collaboration, Search for Physics Beyond the Standard Model in Events with Two Leptons, Jets and Missing Transverse Momentum in pp Collisions at $\sqrt{s}=8$ TeV, JHEP 04 (2015) 124 [arXiv: 1502.06031] [INSPIRE].

[8] N. Vignaroli, Z-peaked excess from heavy gluon decays to vectorlike quarks, Phys. Rev. D 91 (2015) 115009 [arXiv: 1504.01768] [INSPIRE].

[9] U. Ellwanger, Possible explanation of excess events in the search for jets, missing transverse momentum and a $Z$ boson in pp collisions, Eur. Phys. J. C 75 (2015) 367 [arXiv: 1504.02244] [INSPIRE].

[10] B. Allanach, A. Raklev and A. Kvellestad, Consistency of the recent ATLAS $Z+E_{T}^{\text {miss }}$ excess in a simplified GGM model, Phys. Rev. D 91 (2015) 095016 [arXiv:1504.02752] [INSPIRE].

[11] A. Kobakhidze, N. Liu, L. Wu and J.M. Yang, ATLAS Z-peaked excess in the MSSM with a light sbottom or stop, Phys. Rev. D 92 (2015) 075008 [arXiv: 1504.04390] [INSPIRE].

[12] J. Cao, L. Shang, J.M. Yang and Y. Zhang, Explanation of the ATLAS Z-Peaked Excess in the NMSSM, JHEP 06 (2015) 152 [arXiv: 1504.07869] [INSPIRE].

[13] B.A. Dobrescu, Leptophobic Boson Signals with Leptons, Jets and Missing Energy, arXiv: 1506.04435 [INSPIRE].

[14] M. Cahill-Rowley, J.L. Hewett, A. Ismail and T.G. Rizzo, ATLAS Z + MES Excess in the MSSM, Phys. Rev. D 92 (2015) 075029 [arXiv: 1506.05799] [InSPIRE].

[15] X. Lu, S. Shirai and T. Terada, ATLAS Z Excess in Minimal Supersymmetric Standard Model, JHEP 09 (2015) 204 [arXiv: 1506.07161] [INSPIRE].

[16] S.P. Liew, A. Mariotti, K. Mawatari, K. Sakurai and M. Vereecken, Z-peaked excess in goldstini scenarios, Phys. Lett. B 750 (2015) 539 [arXiv:1506.08803] [INSPIRE].

[17] J. Cao, L. Shang, J.M. Yang and Y. Zhang, Explanation of the ATLAS Z-peaked excess by squark pair production in the NMSSM, JHEP 10 (2015) 178 [arXiv:1507.08471] [INSPIRE].

[18] J.H. Collins, J.A. Dror and M. Farina, Mixed Stops and the ATLAS on-Z Excess, Phys. Rev. D 92 (2015) 095022 [arXiv: 1508.02419] [INSPIRE].

[19] R. Ding, Y. Fan, J. Li, T. Li and B. Zhu, The ATLAS Leptonic-Z Excess from Light Squark Productions in the NMSSM Extension with a Heavy Dirac Gluino, arXiv:1508.07452 [INSPIRE].

[20] C.-W. Chiang, K. Harigaya, M. Ibe and T.T. Yanagida, Revisiting R-invariant Direct Gauge Mediation, arXiv:1510.04047 [INSPIRE].

[21] H. Baer, X. Tata and J. Woodside, Phenomenology of Gluino Decays via Loops and Top Quark Yukawa Coupling, Phys. Rev. D 42 (1990) 1568 [InSPIRE].

[22] P. Gambino, G.F. Giudice and P. Slavich, Gluino decays in split supersymmetry, Nucl. Phys. B 726 (2005) 35 [hep-ph/0506214] [INSPIRE]. 
[23] U. Ellwanger, C. Hugonie and A.M. Teixeira, The Next-to-Minimal Supersymmetric Standard Model, Phys. Rept. 496 (2010) 1 [arXiv:0910.1785] [INSPIRE].

[24] K.I. Izawa and T. Yanagida, R invariant natural unification, Prog. Theor. Phys. 97 (1997) 913 [hep-ph/9703350] [INSPIRE].

[25] K. Harigaya, M. Ibe and M. Suzuki, Mass-splitting between haves and have-nots - symmetry vs. Grand Unified Theory, JHEP 09 (2015) 155 [arXiv: 1505.05024] [INSPIRE].

[26] U. Ellwanger, J.F. Gunion and C. Hugonie, NMHDECAY: A Fortran code for the Higgs masses, couplings and decay widths in the NMSSM, JHEP 02 (2005) 066 [hep-ph/0406215] [INSPIRE].

[27] U. Ellwanger and C. Hugonie, NMHDECAY 2.0: An updated program for sparticle masses, Higgs masses, couplings and decay widths in the NMSSM, Comput. Phys. Commun. 175 (2006) 290 [hep-ph/0508022] [INSPIRE].

[28] D. Das, U. Ellwanger and A.M. Teixeira, NMSDECAY: A Fortran Code for Supersymmetric Particle Decays in the Next-to-Minimal Supersymmetric Standard Model, Comput. Phys. Commun. 183 (2012) 774 [arXiv:1106.5633] [INSPIRE].

[29] Y. Giomataris, P. Rebourgeard, J.P. Robert and G. Charpak, MicrOMEGAs: A High granularity position sensitive gaseous detector for high particle flux environments, Nucl. Instrum. Meth. A 376 (1996) 29 [inSPIRE].

[30] G. Bélanger, F. Boudjema, A. Pukhov and A. Semenov, MicrOMEGAs4.1: two dark matter candidates, Comput. Phys. Commun. 192 (2015) 322 [arXiv:1407.6129] [INSPIRE].

[31] M. Drees, H. Dreiner, D. Schmeier, J. Tattersall and J.S. Kim, CheckMATE: Confronting your Favourite New Physics Model with LHC Data, Comput. Phys. Commun. 187 (2014) 227 [arXiv: 1312.2591] [INSPIRE].

[32] DELPHES 3 collaboration, J. de Favereau et al., DELPHES 3, A modular framework for fast simulation of a generic collider experiment, JHEP 02 (2014) 057 [arXiv:1307.6346] [INSPIRE].

[33] M. Cacciari, FastJet: A code for fast $k_{t}$ clustering and more, hep-ph/0607071 [INSPIRE].

[34] M. Cacciari, G.P. Salam and G. Soyez, FastJet User Manual, Eur. Phys. J. C 72 (2012) 1896 [arXiv:1111.6097] [INSPIRE].

[35] J. Alwall, M. Herquet, F. Maltoni, O. Mattelaer and T. Stelzer, MadGraph 5: Going Beyond, JHEP 06 (2011) 128 [arXiv:1106.0522] [INSPIRE].

[36] J. Alwall et al., The automated computation of tree-level and next-to-leading order differential cross sections and their matching to parton shower simulations, JHEP 07 (2014) 079 [arXiv: 1405.0301] [INSPIRE].

[37] T. Sjöstrand, S. Mrenna and P.Z. Skands, PYTHIA 6.4 Physics and Manual, JHEP 05 (2006) 026 [hep-ph/0603175] [INSPIRE].

[38] J. Alwall et al., Comparative study of various algorithms for the merging of parton showers and matrix elements in hadronic collisions, Eur. Phys. J. C 53 (2008) 473 [arXiv: 0706.2569] [INSPIRE].

[39] J. Pumplin, D.R. Stump, J. Huston, H.L. Lai, P.M. Nadolsky and W.K. Tung, New generation of parton distributions with uncertainties from global QCD analysis, JHEP 07 (2002) 012 [hep-ph/0201195] [inSPIRE]. 
[40] https://twiki.cern.ch/twiki/bin/view/LHCPhysics/SUSYCross sections8TeVgluglu.

[41] W. Beenakker et al., NLO+NLL squark and gluino production cross-sections with threshold-improved parton distributions, arXiv:1510.00375 [INSPIRE].

[42] W. Beenakker, R. Hopker, M. Spira and P.M. Zerwas, Squark and gluino production at hadron colliders, Nucl. Phys. B 492 (1997) 51 [hep-ph/9610490] [INSPIRE].

[43] A. Kulesza and L. Motyka, Threshold resummation for squark-antisquark and gluino-pair production at the LHC, Phys. Rev. Lett. 102 (2009) 111802 [arXiv:0807.2405] [INSPIRE].

[44] A. Kulesza and L. Motyka, Soft gluon resummation for the production of gluino-gluino and squark-antisquark pairs at the LHC, Phys. Rev. D 80 (2009) 095004 [arXiv: 0905.4749] [INSPIRE].

[45] W. Beenakker, S. Brensing, M. Krämer, A. Kulesza, E. Laenen and I. Niessen, Soft-gluon resummation for squark and gluino hadroproduction, JHEP 12 (2009) 041 [arXiv: 0909.4418] [INSPIRE].

[46] Planck collaboration, P.A.R. Ade et al., Planck 2015 results. XIII. Cosmological parameters, arXiv:1502.01589 [INSPIRE].

[47] G. Degrassi and P. Slavich, On the radiative corrections to the neutral Higgs boson masses in the NMSSM, Nucl. Phys. B 825 (2010) 119 [arXiv:0907.4682] [INSPIRE].

[48] J.P. Vega and G. Villadoro, SusyHD: Higgs mass Determination in Supersymmetry, JHEP 07 (2015) 159 [arXiv: 1504.05200] [INSPIRE].

[49] T. Hahn, S. Heinemeyer, W. Hollik, H. Rzehak and G. Weiglein, High-Precision Predictions for the Light CP -Even Higgs Boson Mass of the Minimal Supersymmetric Standard Model, Phys. Rev. Lett. 112 (2014) 141801 [arXiv:1312.4937] [INSPIRE].

[50] G. Bélanger, F. Boudjema, C. Hugonie, A. Pukhov and A. Semenov, Relic density of dark matter in the NMSSM, JCAP 09 (2005) 001 [hep-ph/0505142] [INSPIRE].

[51] J.-J. Cao, Z.-X. Heng, J.M. Yang, Y.-M. Zhang and J.-Y. Zhu, A SM-like Higgs near $125 \mathrm{GeV}$ in low energy SUSY: a comparative study for MSSM and NMSSM, JHEP 03 (2012) 086 [arXiv: 1202.5821] [INSPIRE].

[52] K. Ishikawa, T. Kitahara and M. Takimoto, Singlino Resonant Dark Matter and $125 \mathrm{GeV}$ Higgs Boson in High-Scale Supersymmetry, Phys. Rev. Lett. 113 (2014) 131801 [arXiv: 1405.7371] [INSPIRE].

[53] J. Hasenkamp and M.W. Winkler, NMSSM with Gravitino Dark Matter to be tested at LHC, Nucl. Phys. B 877 (2013) 419 [arXiv: 1308.2678] [INSPIRE].

[54] LUX collaboration, D.S. Akerib et al., First results from the LUX dark matter experiment at the Sanford Underground Research Facility, Phys. Rev. Lett. 112 (2014) 091303 [arXiv: 1310.8214] [INSPIRE].

[55] LUX collaboration, D.S. Akerib et al., The Large Underground Xenon (LUX) Experiment, Nucl. Instrum. Meth. A 704 (2013) 111 [arXiv:1211.3788] [INSPIRE].

[56] XENON1T collaboration, E. Aprile, The XENON1T Dark Matter Search Experiment, Springer Proc. Phys. 148 (2013) 93 [arXiv:1206.6288] [InSPIRE].

[57] XMASS collaboration, K. Hiraide, XMASS: Recent results and status, arXiv:1506.08939 [INSPIRE]. 
[58] DARKSIDE collaboration, E. Pantic, The DarkSide-G2 experiment at LNGS, Talk given UCLA's 11th Symposium on Sources and Detection of Dark Matter and Dark Energy in the Universe, Northwest Campus Auditorium at Covel Commons, UCLA Los Angeles, U.S.A., February 26-28, 2014.

[59] J. Hajer, Y.-Y. Li, T. Liu and J.F.H. Shiu, Heavy Higgs Bosons at 14 TeV and 100 TeV, JHEP 11 (2015) 124 [arXiv: 1504.07617] [INSPIRE].

[60] A. Arbey, M. Battaglia and F. Mahmoudi, Supersymmetric Heavy Higgs Bosons at the LHC, Phys. Rev. D 88 (2013) 015007 [arXiv: 1303.7450] [InSPIRE].

[61] T. Li, Decoupling MSSM Higgs Sector and Heavy Higgs Decay, Phys. Lett. B 728 (2014) 77 [arXiv: 1309.6713] [INSPIRE].

[62] ATLAS collaboration, Search for a high-mass Higgs boson decaying to a $W$ boson pair in pp collisions at $\sqrt{s}=8$ TeV with the ATLAS detector, arXiv:1509.00389 [INSPIRE].

[63] ATLAS collaboration, A search for $t \bar{t}$ resonances using lepton-plus-jets events in proton-proton collisions at $\sqrt{s}=8$ TeV with the ATLAS detector, JHEP 08 (2015) 148 [arXiv: 1505.07018] [INSPIRE].

[64] M.J. Dolan, C. Englert and M. Spannowsky, New Physics in LHC Higgs boson pair production, Phys. Rev. D 87 (2013) 055002 [arXiv:1210.8166] [INSPIRE].

[65] J.M. No and M. Ramsey-Musolf, Probing the Higgs Portal at the LHC Through Resonant di-Higgs Production, Phys. Rev. D 89 (2014) 095031 [arXiv:1310.6035] [INSPIRE].

[66] C.-Y. Chen, S. Dawson and I.M. Lewis, Exploring resonant di-Higgs boson production in the Higgs singlet model, Phys. Rev. D 91 (2015) 035015 [arXiv:1410.5488] [INSPIRE].

[67] V. Martín Lozano, J.M. Moreno and C.B. Park, Resonant Higgs boson pair production in the $h h \rightarrow b \bar{b} W W \rightarrow b \bar{b} \ell^{+} \nu \ell^{-} \bar{\nu}$ decay channel, JHEP 08 (2015) 004 [arXiv:1501.03799] [INSPIRE].

[68] S. Dawson and I.M. Lewis, NLO corrections to double Higgs boson production in the Higgs singlet model, Phys. Rev. D 92 (2015) 094023 [arXiv:1508.05397] [INSPIRE]. 